\title{
Medicamentos potencialmente inadequados utilizados no tratamento do diabetes em idosos: uma revisão integrativa*
}

\section{Potentially inappropriate medications used in the diabetes treatment in older patients: an integrative review}

\author{
Isabella Ribeiro Silva ${ }^{1}$ Isabella de Figueiredo Zuppo ${ }^{2} \bullet$ Mirella Monique Lana Diniz ${ }^{3} \bullet$ \\ Mariana Martins Gonzaga do Nascimento ${ }^{4}$
}

\begin{abstract}
RESUMO
Objetiva-se avaliar as evidências acerca do perfil de segurança para idosos dos medicamentos utili-zados no tratamento do Diabetes Mellitus tipo 2, que são considerados medicamentos potencialmen-te inadequados pelo critério de Beers e pela ferramenta STOPP: glibenclamida e clorpropamida. Realizou-se uma revisão integrativa da literatura realizada a partir da base de dados PubMed. Foram utilizados os descritores do banco Medical Subject Headings (MeSH) "chlorpropamide", "glyburide"e "sulfonylureas" combinados ao descritor "aged".Foram incluídos artigos que avaliaram o perfil de segurança do uso de clorpropamida e/ou glibenclamida por idosos. Sete artigos foram selecionados. Não foi encontrado nenhum estudo que avaliasse a clorpropamida. Dois estudos avaliaram a segu-rança cardiovascular e o risco de morte com glibenclamida, mas não foram apontadas diferenças entre o uso deste medicamento e de outros da classe das sulfonilureias. Os demais trabalhos eviden-ciaram uma associação entre o uso de glibenclamida e hipoglicemia, apesar de apresentarem resultados conflitantes quanto à relação da ocorrência deste evento com a função renal dos pacientes ido-sos. Concluí-se que a literatura aponta a necessidade de reavaliação da prescrição de glibenclamida, dada sua comprovada relação com o desenvolvimento de hipoglicemia em pacientes idosos.
\end{abstract}

Palavras-chave: Diabetes Mellitus; Idoso; Glibenclamida; Clorpropamida; Efeitos Adversos.

\begin{abstract}
The aim is to evaluate scientific evidences about the safety profile among older patients of drugs used to treat type 2 Diabetes that are considered potentially inappropriate medications by the Beers criteria and STOPP: glyburide and chlorpropamide. An integrative review was performed on Pub-Med database using Medical Subject Headings (MeSH), using the descriptors: "chlorpropamide","glyburide","sulfonylureas" and "aged". Articles that evaluated the safety profile of chlorpropamide and/or glyburide for older patients were included. Seven articles were selected, none of which as-sessed the safety profile of chlorpropamide. Two studies evaluated the cardiovascular safety and the risk of death with glyburide, but no difference between this product and other drugs from the sul-fonylureas class was identified. The other studies showed an association between the use of gly-buride and hypoglycemia, despite showing conflicting results regarding the relationship between this event and renal function of older patients. It concludes that the scientific literature showed the needing for reassessment of glyburide prescription among older patients, given its proven relation-ship with the occurrence of hypoglycemia.
\end{abstract}

Keywords: Diabetes Mellitus; Aged; Glyburide; Chlorpropamide; Adverse Effects.

NOTA

1. Especialista em Farmacologia Clínica pela Associação Mineira de Farmacêuticos/Centro Universitário Newton Pai-va; Mestranda em Ciências da Saúde pela Universidade Federal de São João del-Rei

2. Farmacêutica do Hospital Materdei

3. Graduanda em Medicina pela Universidade Federal de Minas Gerais

4. Doutora em Ciências da Saúde pelo Centro de Pesquisas René Rachou/Fundação Oswaldo Cruz; Pós-doutoranda em Medicamentos e Assistência Farmacêutica pela Faculdade de Farmácia da Universidade Federal de Minas Gerais.

* Trabalho baseado em Trabalho de Conclusão de Curso em Farmacologia Clínica pela Associação Mineira de Far-macêuticos/Centro Universitário Newton Paiva da primeira autora. Belo Horizonte/MG, 2015. 


\section{INTRODUÇÃO}

O envelhecimento da população vem ocorrendo em todo o mundo e recentemente de forma mais acentuada nos países em desenvolvimento ${ }^{1}$. Estas alterações observadas na distribuição etária populacional são consequência da expressiva redução da natalidade, fecundidade e mortalidade, somadas ao notável avanço tecnológico e às ações e políticas adotadas pela sociedade e pelo Estado nos últimos anos ${ }^{2-3}$.

O aumento da longevidade e expectativa de vida, apesar de soarem como indicadores positivos, não necessariamente refletem melhorias diretas na qualidade de vida e condições de saúde do indivíduo ${ }^{4}$. A população idosa, em geral, apresenta doenças crônicas e comorbidades que demandam acompanhamento e cuidado constantes, além de farmacoterapia múltipla e contínua².

O envelhecimento da população, associada a outros fatores ambientais e genéticos, é responsável, em grande parte, pela alta prevalência de doenças crônicodegenerativas, sendo o Diabetes Mellitus (DM) uma delas ${ }^{5}$. Estima-se que 382 milhões de indivíduos possuam a doença no mundo, dos quais 134,6 milhões são idosos com idade entre 60 e 79 anos. 0 Brasil é o quarto país com maior número de casos de DM em adultos, totalizando 11,9 milhões de pessoas com a doença ${ }^{6}$. A prevalência entre a população idosa no país chega a $14,6 \% \%^{7-8}$. A previsão é que haja um crescimento anual de $2,2 \%$ de casos em todo o mundo, com um aumento absoluto de $54 \%$ até 2030 , o que representa praticamente o dobro das taxas de crescimento da população adulta mundial? .

O tratamento medicamentoso do Diabetes Mellitus tipo 2 (DM2) em idosos apresenta maior complexidade, tendo em vista as condições de saúde e os fatores de risco associados à idade, tais como polifarmácia, comprometimento visual e cognitivo, disfunção renal, maior risco de quedas, entre outros fatores ${ }^{10}$. Considerando estas particularidades, foram conduzidos estudos e desenvolvidas ferramentas que visam identificar e classificar medicamentos potencialmente inadequados (MPI) em idosos.

O primeiro trabalho a abordar a farmacoterapia no idoso sob esse aspecto foi o critério desenvolvido por Beers et al. (1991) para aplicação em instituições de longa permanência ${ }^{11}$. Desde sua publicação, o critério tem sido revisado e adaptado para ter aplicabilidade também em outras circunstâncias e ambientes. Atualmente, o documento é dividido em seis listas: (I) MPI para idosos; (II) MPI para idosos devido a interações com determinadas síndromes ou doenças; (III) medicamentos que devem ser utilizados com cautela em idosos; (IV) lista de interações medicamentosas que devem ser evitadas em idosos; (V) medicamentos que devem ser evitados ou ter sua dose reduzida em idosos com disfunção renal; e (VI) medicamentos com propriedades anticolinérgicas pronunciadas ${ }^{(12)}$. Sua relevância na rotina clínica, entretanto, é questionável e novas ferramentas têm sido desenvolvidas para abranger pontos críticos que não são considerados no critério de Beers. Uma delas é a ferramenta Screening Tool of Older Persons' Prescriptions (STOPP), que selecionou medicamentos ou classes terapêuticas e as classificou como prescrições potencialmente inadequadas para idosos ${ }^{13}$. Apesar de algumas divergências entre os critérios, ambos apontam medicamentos comumente utilizados no tratamento da DM2 em idosos como MPI: glibenclamida e clorpropamida ${ }^{10}$.

Tendo em vista a crescente prevalência de DM em idosos e a complexidade da farmacoterapia nessa população, o presente estudo tem como objetivo realizar uma revisão de literatura sobre o perfil de segurança da glibenclamida e clorpropamida para a população idosa.

\section{MÉTODOS}

O presente estudo é uma revisão integrativa realizada com base em fontes secundárias de informação. Para tal, foi realizada uma busca no PubMed, da biblioteca Medical Literature Analysis and Retrieval System on Line (Medline), na qual é disponibilizada pela National Library of Medicine e pelo National Institute of Health dos Estados Unidos da América.

Em um primeiro momento, os descritores MeSH "chlorpropamide", "glyburide" e "sulfonylureas" foram combinados ao descritor MeSH "aged" com utilização do operador boleano "AND". Também foi utilizado como estratégia de busca o uso individual dos descritores "chlorpropamide", "glyburide" e "sulfonylureas" adicionandose o limite de estudos em indivíduos com 65 anos ou mais. Para todas as buscas, foram utilizados os seguintes limites: estudos realizados em humanos, publicados de 2004 a 2014, e nos idiomas inglês, português ou espanhol. As buscas foram realizadas em dezembro de 2014.

A seleção inicial dos artigos foi realizada por dois pesquisadores independentes após análise do título e resumo dos artigos recuperados nas buscas. Os artigos assim selecionados foram lidos na íntegra, sendo julgado o seu atendimento aos critérios de inclusão e exclusão, os quais foram: 1) inclusão - artigos que avaliaram o perfil de segurança do uso de clorpropamida e/ou glibenclamida por idosos; e 2) exclusão - comentários, relatos de caso ou artigos cuja amostra estudada incluía indivíduos com idade inferior a 60 anos e não propunham análise de dados seccionada por faixa etária. No caso de discordância entre os pesquisadores, a permanência de um artigo neste estudo foi decidida por um terceiro avaliador, observando-se os critérios de inclusão e exclusão definidos para esta revisão.

Os trabalhos selecionados foram analisados com foco na recuperação de informações sobre a ocorrência de eventos adversos relacionados ao uso de clorpropamida ou glibenclamida por idosos.

\section{RESULTADOS}

No total, foram recuperados 947 artigos. Destes, apenas 30 foram selecionados para leitura na íntegra, dos quais 7 
foram incluídos na seleção final, após exclusão detalhada na figura 1.

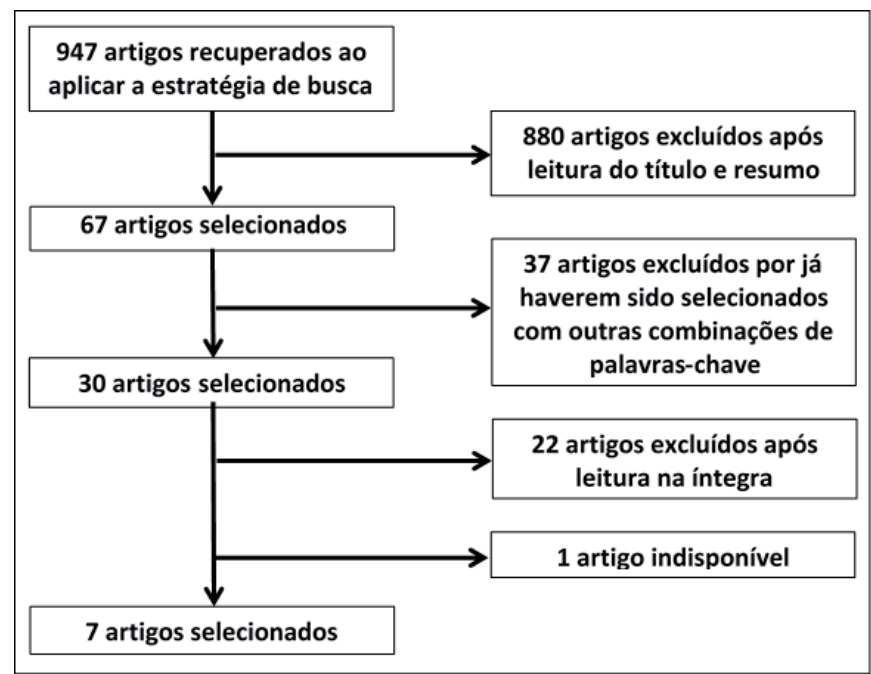

Figura 1 - Fluxograma de pesquisa e seleção de artigos científicos sobre a segurança de medica-mentos potencialmente inadequados para idosos utilizados no tratamento do Diabetes Mellitus. Belo Horizonte, MG, Brasil, 2014.

Fonte: dados da própria pesquisa.

Todos os artigos incluídos analisaram a segurança do uso de glibenclamida em relação a outras sulfonilureias ou outras classes de antidiabéticos orais, mas nenhum deles avaliou a segurança da clorpropramida. 0 número de artigos recuperados a partir de casa uma das palavraschave ou combinações destas está relacionado na tabela 1.

\section{Glibenclamida e segurança cardiovascular}

Dos 7 estudos selecionados, 2 abordaram o perfil de segurança cardiovascular da glibenclamida. 0 estudo de Juurlink et al. acompanhou por um período de 4 anos uma coorte composta por todos os idosos com 66 anos ou mais, admitidos em instituições hospitalares de Ontário (Canadá), em decorrência de infarto agudo do miocárdio (IAM) e/ou para intervenção coronária percutânea; e que apresentavam uma prescrição de glibenclamida ou gliclazida nos 90 dias que antecederam a internação ${ }^{15}$.
Os autores não encontraram diferenças estatisticamente significantes no risco morte, incidência de IAM, insuficiência cardíaca ou morte com gliblenclamida em comparação à gliclazida (hazard ratio ajustada 1.01; 95\% IC 0,86-1,18). A análise isolada de cada um dos desfechos também não retornou diferenças significantes.

Andersson et al. avaliaram a mortalidade entre 3.477 indivíduos dinamarqueses com idade entre 65 e 87 anos, hospitalizados em decorrência de insuficiência cardíaca, e que utilizavam sulfonilureia (glibenclamida, glipizida, gliclazida e tolbutamida) em monoterapia ${ }^{16}$. Não foi observada diferença no risco de morte entre os usuários de glibenclamida em relação às demais sulfonilureias.

\section{Glibenclamida e Hipoglicemia}

Um estudo randomizado aberto conduzido com 88 idosos (65 anos ou mais) avaliou a segurança da repaglinida em comparação à glibenclamida quanto à ocorrência de hipoglicemia (glicemia inferior a $4 \mathrm{mmol} / \mathrm{L}$ ) e outros eventos adversos na população estudada. A proporção de pacientes que apresentaram pelo menos um episódio de hipoglicemia foi de $26,1 \%$ com repaglinida e $42,0 \%$ com glibenclamida $(p=0,026)$. Entre indivíduos com mais de 75 anos, ocorreram 13 episódios de hipoglicemia com o uso da repaglinida e 31 episódios com glibenclamida $(p=$ 0,04). 0 número de episódios de hipoglicemia por pacientetempo também foi menor com repaglinida do que com a sulfonilureia $(p=0,002)^{17}$.

O trabalho de Meneilly também avaliou o uso de repaglinida e glibenclamida em 13 idosos, e sua associação com o perfil insulínico e glicêmico destes indivíduos ${ }^{18}$. Neste estudo, não foi observada diferença estatisticamente significativa na área sob a curva do perfil plasmático de insulina entre 0 e 240 minutos após uma refeição padronizada entre os dois grupos $(p>0,05)$. No entanto, nos primeiros 30 minutos, indivíduos sob uso de glibenclamida apresentaram menores níveis plasmáticos de insulina $(p<0,01)$, e o contrário foi observado no perfil de insulina plasmática medida no tempo de 180 a 240 minutos $(p<0,01)$. Cinco pacientes tratados com

Tabela 1 - Número de artigos recuperados de acordo com o descritor ou combinações de descritores. Belo Horizonte, MG, Brasil, 2014.

\begin{tabular}{l|c|c|c|c|c}
\hline Combinação de descritores & $\begin{array}{c}\text { Artigos } \\
\text { recuperados }\end{array}$ & $\begin{array}{c}\text { Artigos } \\
\text { selecionados } \\
\text { para leitura na } \\
\text { íntegra }\end{array}$ & $\begin{array}{c}\text { Artigos } \\
\text { selecionados em } \\
\text { busca anterior }\end{array}$ & $\begin{array}{c}\text { Artigos lidos na } \\
\text { íntegra }\end{array}$ & $\begin{array}{c}\text { Artigos não } \\
\text { incluídos nos } \\
\text { resul-tados }\end{array}$ \\
selecionados \\
\hline "chlorpropamide" AND "aged" & 2 & 0 & 0 & 0 & 0 \\
\hline "chlorpropamide"* & 2 & 0 & 0 & 0 & 0 \\
\hline "glyburide" AND "aged" & 154 & 11 & 0 & 11 & 5 \\
\hline "glyburide"* & 154 & 8 & 8 & 0 & 0 \\
\hline "sulfonylureas" AND "aged" & 635 & 24 & 3 & 21 & 20 \\
\hline "sulfonylureas"* & 635 & 24 & 24 & 0 & 0 \\
\hline
\end{tabular}

Fonte: dados da própria pesquisa. 
glibenclamida apresentaram hipoglicemia (glicose plasmática $<3,5 \mathrm{mmol}$ ) durante a condução do estudo, ao passo que em nenhum dos indivíduos tratados com repaglinida foi observado este evento ${ }^{18}$.

Weir et al. realizaram um estudo caso-controle no qual a ocorrência de hipoglicemia associada à metformina e à glibenclamida foi comparado. Também foi avaliada a influência da função renal dos idosos (65 anos ou mais) frente a esse risco ${ }^{19}$. Casos foram definidos como admissão hospitalar devido ao diagnóstico de hipoglicemia, e para cada caso foi selecionado um controle aleatório com equivalência de sexo e idade $( \pm 1$ ano) sem evidência de hipoglicemia registrada. Os grupos caso e controle foram divididos de acordo com a função renal - normal ou alterada (Taxa de Filtração Glomerular (TFG) $\geqslant$ ou $<60$ $\mathrm{mL} / \mathrm{min} / 1,73 \mathrm{~m}^{2}$, respectivamente). Entre os pacientes com função renal normal, foi identificada uma chance nove vezes maior de ocorrência de hipoglicemia grave com o uso de glibenclamida (OR 9,0, 95\% IC 4,9-16,4). Os autores constataram ainda que a função renal não modifica significativamente essa chance $(p=0,15)^{19}$.

Aspinall et al. avaliaram o impacto de uma estratégia de incentivo à descontinuação do tratamento com glibenclamida entre idosos com creatinina sérica elevada no sistema de saúde de assistência aos veteranos dos Estados Unidos (Veterans Integrated Service Networks) ${ }^{20}$. A troca ou suspensão do uso de glibenclamida foi adotada em $71,5 \%$ dos pacientes idosos com alteração renal e $56 \%$ daqueles sem alteração renal. Não foi detectada diferença estatisticamente significativa nos níveis de hemoglobina glicada (7,17 vs 7,22\%; $p=0,36)$ ou na taxa de incidência de hipoglicemia por pessoa-dia (0,093 vs 0,$070 ; p=0,10$ ) antes e após a suspensão de glibenclamida ou troca por outro medicamento. No entanto, realizando a análise com estratificação de grupos de acordo com os níveis de creatinina sérica, detectou-se que aqueles idosos com creatinina sérica superior (creatinina plasmática $\geqslant 3 \mathrm{mg} / \mathrm{dL}$ ) apresentavam maior taxa de incidência de hipoglicemia por paciente-dia antes da suspensão ou troca da glibenclamida $(0,169 \text { vs 0,039; } p=0,003)^{20}$.

Em estudo prospectivo que envolvia 99 indivíduos com idade igual ou superior a 80 anos, Greco et al. avaliaram a incidência de hospitalização devido à hipoglicemia e detectaram que, entre os usuários de tratamento oral para - DM, a maioria dos pacientes com hipoglicemia grave eram usuários de glibenclamida (76,8\%), com destaque para aqueles sob uso de glibenclamida isolada $(53,5 \%)^{21}$.

\section{DISCUSSÃO}

Apesar dos impactos do tratamento medicamentoso do DM2 em idosos, ainda são escassos os estudos que avaliam o perfil de segurança dos MPI - clorpropamida e glibenclamida - utilizados no tratamento da doença nessa população. Estes agentes ainda são prescritos e utilizados entre idosos no Brasil apesar de serem apontados como MPI desde as primeiras versões do critério de Beers e da ferramenta STOPP ${ }^{22}$. Isso destaca a necessidade de divulgarmos a temática sobre o grupo de MPI em um país com uma população crescente de idosos e com demanda considerável por profissionais qualificados e atualizados para seu cuidado.

O fato de não haver estudos que abordem a segurança do uso da clorpropamida por idosos pode ser explicado pelo seu desuso na prática clínica, uma vez que alternativas terapêuticas com similar efetividade e segurança superior estão disponíveis. O medicamento já não consta nas diretrizes terapêuticas internacionais porém ainda é citado no documento elaborado pela Sociedade Brasileira de Diabetes, onde é destacado o risco de hipoglicemia e hiponatremia quando utilizado por idosos, sendo seu uso não recomendado nesta população ${ }^{14-23-25}$. Apesar disso, um número considerável de estudos ainda apontam a prescrição da clorpropamida entre indivíduos com mais de 60 anos na população brasileira ${ }^{26-28}$. Dados alertam para a prática irracional de prescrição entre idosos diabéticos e evidenciam a exposição destes pacientes ao risco de hipoglicemia, que apresenta alta prevalência entre idosos e consequências graves como quedas, fraturas, dano cognitivo, admissões hospitalares entre outras ${ }^{29-31}$.

A glibenclamida, quando avaliada quanto ao risco de morte e à sua relação com a incidência de IAM e/ ou intervenção coronária percutânea em idosos, não apresentou diferença estatisticamente significativa em relação às outras sulfonilureias. Nos trabalhos selecionados, a comparação só foi realizada entre medicamentos desta classe terapêutica. Não foram encontrados estudos que comparassem a segurança cardiovascular e o risco de morte da glibenclamida em relação a outros antidiabéticos orais de diferentes classes utilizados no tratamento do DM2.

Entre os estudos analisados, a maioria aponta a associação entre a utilização de glibenclamida e a ocorrência de hipoglicemia. Resultados conflitantes aparecem quando se avalia a ocorrência de hipoglicemia associada à função renal dos pacientes idosos. Mesmo sendo uma reação adversa esperada para este medicamento, considerando seu mecanismo de ação e elevado tempo de meia-vida, os resultados destes estudos permitem mensurar a frequência da hipoglicemia entre indivíduos idosos, sobretudo quando comparada a outros medicamentos de uso oral para o tratamento de DM2. A segurança comparativa destaca a desvantagem do uso da glibenclamida em relação a representantes de outras classes terapêuticas, como a metformina e a repaglinida, apesar dos estudos comparativos diretos com a repaglinida apresentarem uma amostra reduzida de idosos.

De uma forma geral, as diretrizes nacionais e internacionais fazem uma abordagem semelhante do tratamento do DM2. Especificamente para os agentes hipoglicemiantes orais, a metformina sempre aparece como primeira linha de tratamento, sendo associada, se necessário, a uma sulfonilureia. Segundo a diretriz da International Diabetes Federation ${ }^{31}$, as sulfonilureias 
são contraindicadas em pacientes com risco elevado de hipoglicemia, ou naqueles em que a hipoglicemia implica em consequências mais graves, como os idosos. Tal contraindicação é reforçada pela International Association of Gerontology and Geriatrics ${ }^{29}$. As diretrizes tanto da American Diabetes Association quanto da American Geriatrics Society ressaltam a ação hipoglicemiante das sulfonilureias e excluem a glibenclamida e a clorpropramida do grupo de alternativas terapêuticas dessa classe para o tratamento de DM2 em idosos ${ }^{14-32}$. A American Geriatrics Society recomenda o uso de insulina no lugar destes secretagogos ${ }^{32}$.

No entanto, a diretriz da Sociedade Brasileira de Diabetes traz as sulfonilureias, inclusive glibenclamida e clorpropramida, como alternativas terapêuticas de segunda linha no tratamento da DM2, ou em associação à metformina. 0 documento esclarece que, para os idosos, não se deve prescrever a clorpropramida, pelo risco de hipoglicemia e hiponatremia; e que glicazida e glimepirida devem ser preferidas à glibenclamida, por estarem associadas a risco de hipoglicemia ${ }^{24-25}$.

Ademais, diretrizes para manejo do DM2 indicam que o controle da glicemia nos idosos não deve ser tão rigoroso quanto o realizado para indivíduos mais jovens, sendo aceitável que a hemoglobina glicada (HbA1C) fique entre 7,0 e 7,5\% ou até em níveis mais elevados (7,5-9\%) em idosos frágeis, com múltiplas comorbidades e baixa expectativa de vida, diminuindo, assim, os riscos de indução de hipoglicemia ${ }^{29-31-32}$. Também é ressaltada a necessidade da equipe de saúde estar preparada para lidar com o tratamento de pacientes idosos com DM2, uma vez que o manejo dessa doença neste grupo de pacientes depende de conhecimentos e habilidades específicas em função de suas características fisiopatológicas que podem alterar a farmacocinética e farmacodinâmica dos medicamentos utilizados para tratá-la.

\section{CONCLUSÃO}

O objetivo dessa revisão foi alcançado uma vez que os estudos recuperados apresentam dados sobre o perfil de segurança dos medicamentos de uso oral para o tratamento do DM2 considerados MPI para idosos, segundo o critério de Beers e a ferramenta STOPP \& START.

Os estudos recuperados destacaram a necessidade de reavaliação da prescrição da glibenclamida, dada sua comprovada relação com o desenvolvimento de hipoglicemia em pacientes idosos. A ausência de estudos sobre a segurança da clorpropamida refletem o perfil obsoleto deste medicamento na terapia do diabetes e reforçam a criticidade da sua utilização.

Desta forma, o presente estudo cumpre o importante papel de divulgar informações acerca deste grupo de MPI que causa mais risco que benefícios em uma população crescente que é a geriátrica portadora de diabetes e ressaltar a necessidade de atualização constante dos profissionais de saúde no tocante a esta área.

\section{REFERÊNCIAS}

1. Lima-Costa MF, Veras R. Saúde pública e envelhecimento. Cad Saúde Pública [Internet]. 2003; 19(3):700-1. Disponível em: http://dx.doi.org/10.1590/S0102-311X2003000300001.

2. Closs VE, Schwanke CHA. A evolução do índice de envelhecimento no Brasil, nas suas regiões e unidades federativas no período de 1970 a 2010. Rev Bras Geriatr Gerontol [Internet]. 2012; 15(3):443-58. Disponível em: http://dx.doi.org/10.1590/S1809-98232012000300006.

3. Vasconcelos AMN, Gomes MMF. Transição demográfica: a experiência brasileira. Epidemiol Serv Saúde [Internet]. 2012; 21(4):539-48. Disponível em: http://dx.doi.org/10.5123/ S1679-49742012000400003.

4. Lima-costa MF, Matos DL, Camargos VP, Macinko J. Tendências em dez anos das condições de saúde de idosos brasileiros: evidências da Pesquisa Nacional por Amostra de Domicílios (1998, 2003, 2008). Ciênc Saúde Coletiva [Internet]. 2011; 16(9):3689-96. Disponível em: http://dx.doi.org/10.1590/ S1413-81232011001000006.

5. Duncan BB, Chor D, Aquino EML, Bensenor IM, Mill JG, Schimidt MI, Lotufo PA, Vigo A, Barreto SM. Doenças Crônicas Não Transmissíveis no Brasil: prioridade para enfrentamento e investigação. Rev Saúde Pública [Internet]. 2012; 46(supl 1):126-34. Disponível em: http://dx.doi.org/10.1590/S003489102012000700017.

6. International diabetes federation. IDF Diabetes Atlas [Internet]. 6 ed. IDF, 2013. 159p. Disponível em: www. diabetesatlas.org.

7. Passos VMA, Barreto SM, Diniz LM, Lima-Costa MF. Type 2 diabetes: prevalence and associated factors in a Brazilian community - the Bambuí health and aging study [Internet]. São Paulo Med J. 2005; 123(2):66-71.Disponível em: http:// dx.doi.org/10.1590/S1516-31802005000200007.

8. Lebrão ML, Duarte YAO. O projeto SABE no município de São Paulo: uma abordagem inicial. Brasília: Organização PanAmericana de Saúde; 2003. 255 p.

9. Wild S, Roglic G, Green A, Sicree R, King H. Global Prevalence of Diabetes: estimates for the year 2000 and projections for 2030. Diabetes Care [Internet]. 2004; 27(5):1047-53. Disponível em: https://doi.org/10.2337/diacare.27.5.1047.

10. Fravel MA, McDanel DL, Ross MB, Moores KG, Starry MJ. Special considerations for treatment of type 2 diabetes mellitus in the elderly. Am J Health-Syst Pharm [Internet]. 2011; 68(6):500-9. Disponível em: http://dx.doi.org/10.2146/ajhp080085.

11. Beers MH, Ouslander JG, Rollingher I, Reuben DB Brooks J, Beck JC. Explicit criteria for determining inappropriate medication use in nursing home residents. UCLA Division of Geriatric Medicine. Arch Intern Med [Internet]. 1991; 151(9):1825-32. Disponível em: http://dx.doi.org/10.1001/ archinte.1991.00400090107019.

12. American Geriatrics Society. American Geriatrics Society 2015 Beers Criteria Update Expert Panel. American Geriatrics Society 2015 Updated Beers Criteria for Potentially Inappropriate Medication Use in Older Adults. J Am Geriatr Soc [Internet]. 2015; 63(11):2227-46. Disponível em: http:// dx.doi.org/10.1111/jgs.13702.

13. O'Mahony D. O'Sullivan D, Byrne S, O'Connor MN, Ryan C, Gallagher P. STOPP/START criteria for potentially 
inappropriate prescribing in older people: version 2. Age Ageing [Internet]. 2015; 44(2):213-8. Disponível em: http:// dx.doi.org/10.1093/ageing/afu145.

14. American Diabetes Association. Standards of Medical Care in Diabetes - 2014. Diabetes Care [Internet]. 2014; 37(supl 1):S14-S80. Disponível em: http://dx.doi.org/10.2337/ dc14-S014.

15. Juurlink DN, Gomes T, Shah BR, Mamdani MM. Adverse cardiovascular events during treatment with glyburide (glibenclamide) or gliclazide in a high-risk population. Diabet Med [Internet]. 2012; 29(12):1524-8. Disponível em: http://dx.doi.org/10.1111/j.1464-5491.2012.03772.x.

16. Andersson C, Gislason $\mathrm{GH}$, Jorgensen $\mathrm{CH}$, Hansen PR, Vaag A, Sorensen R, et al. Comparable long-term mortality risk associated with individual sulfonylureas in diabetes patients with heart failure. Diabetes Res Clin Pract [Internet]. 2011; 94(1):119-25. Disponível em: http://dx.doi.org/10.1016/j. diabres.2011.07.011.

17. Papa G, Fedele V, Rizzo MR, Fioravanti M, Leotta C, Solerte SB, Purello F, Paolisso G. Safety of Type 2 Diabetes Treatment With Repaglinide Compared With Glibenclamide in Elderly People. Diabetes Care [Internet]. 2006; 29(8):1918-20. Disponível em: http://dx.doi.org/10.2337/dc05-2495.

18. Meneilly GS. Effect of Repaglinide Versus Glyburide on Postprandial Glucose and Insulin Values in Elderly Patients with Type 2 Diabetes. Diabetes Technol Ther [Internet]. 2011; 13(1):63-5. Disponível em: http://dx.doi.org/10.1089/ dia.2010.0105.

19. Weir MA, Gomes T, Mamdani M, Juurlink DN, Hackam DG, Mahon JL, Jain AK, Garg AX. Impaired renal function modifies the risk of severe hypoglycemia among users of insulin but not glyburide: a population-based nested case-control study. Nephrol Dial Transplant [Internet]. 2011; 26(6):188894. Disponível em: http://dx.doi.org/10.1093/ndt/gfa649.

20. Aspinall SL, Zhao X, Good CB, Stone RA, Boresi J, Bartholomew C, et al. Intervention to Decrease Glyburide Use in Elderly Patients With Renal Insufficiency. Am J Geriatr Pharmacother [Internet]. 2011; 9(1):58-68. Disponível em: http://dx.doi. org/10.1016/j.amjopharm.2011.02.001.

21. Greco D, Pisciotta M, Gambina F, Maggio F. Severe Hypoglycaemia Leading to Hospital Admission in Type 2 Diabetic Patients Aged 80 Years or Older. Exp Clin Endocrinol Diabetes [Internet]. 2010; 118(4):215-19. Disponível em: http://dx.doi.org/10.1055/s-0029-1241823.

22. Nascimento MMG, Lima-Costa MF, Loyola Filho Al. Potentially Inappropriate Medication Use Among Brazilian Elderly: A Population-Based Pharmacoepidemiological Study. Acta Farm Bonaerense. 2015; 35(4):659-66.

23. European Society of Cardiology. ESC Guidelines on diabetes, pre-diabetes, and cardiovascular diseases developed in collaboration with the EASD - Summary. Diab Vasc Dis Res [Internet]. 2014; 11(3):133-73. Disponível em: http://dx.doi. org/10.1177/1479164114525548.

24. Sociedade Brasileira De Diabetes. Diretrizes da Sociedade Brasileira de Diabetes 2014-2015 [Internet]. 2015; 374 p. Disponível em: http://www.diabetes.org.br.

25. Sociedade Brasileira De Diabetes. Conduta Terapêutica no Diabetes tipo 2: algoritmo SBD 2015 [Internet]. 2015; 36 p.Disponível em: http://www.diabetes.org.br.
26. Ribas C, Oliveira KR. Perfil dos medicamentos prescritos para idosos em uma Unidade Básica de Saúde do município de Ijuí-RS. Rev Bras Geriatr Gerontol [Internet]. 2014; 17(1):99114. Disponível em: http://dx.doi.org/10.1590/S180998232014000100011.

27. Gontijo MF, Ribeiro AQ, Klein CH, Rozenfeld S, Acurcio FA. Uso de anti-hipertensivos e antidiabéticos por idosos: inquérito em Belo Horizonte, Minas Gerais, Brasil. Cad. Saúde Pública [Internet]. 2012; 28(7):1337-46. Disponível em: http://dx.doi. org/10.1590/S0102-311X2012000700012.

28. Gautério DP,Santos SSC,Teda-Pelzer MT,Barros EJ,Baumgarten L. Caracterização dos idosos usuários de medicação residentes em instituição de longa permanência. Rev Esc Enferm USP [Internet]. 2012; 46(6):1394-99. Disponível em: http://dx.doi.org/10.1590/S0080-62342012000600016.

29. Sinclair A, Morley JE, Rodriguez-Manas L, Paolisso G, Bayer T, Zeyfang A, et al. Diabetes mellitus in older people: position statement on behalf of the International Association of Gerontology and Geriatrics (IAGG), the European Diabetes Working Party for Older People (EDWPOP), and the International Task Force of Experts in Diabetes. J Am Med Dir Assoc [Internet]. 2012; 13(6):497-502. Disponível em: http:// dx.doi.org/10.1016/j.jamda.2012.04.012.

30. Feinkohl I, Aung PP, Keller M, Robertson CM, Morling JR, McLachlan S, et al. Severe Hypoglycemia and Cognitive Decline in Older People With Type 2 Diabetes: The Edinburgh Type 2 Diabetes Study. Diabetes Care [Internet]. 2014; 37(2):507-15. Disponível em: http://dx.doi.org/0.2337/dc13-1384.

31. International Diabetes Federation. Managing older people with type 2 diabetes. IDF [Internet]. 2013. 94 p. Disponível em: http://www.idf.org/guidelines-older-people-type-2diabetes.

32. America Geriatrics Society. Guidelines abstracted from the American Geriatrics Society Guidelines for Improving the Care of Older Adults with Diabetes Mellitus: 2013 update. J Am Geriatr Soc [Internet]. 2013; 61(11):2020-6. Disponível em: http://dx.doi.org/10.1111/jgs.12514. 